\title{
SEMICLASSICAL APPROXIMATION IN A REALISTIC ONE-BODY POTENTIAL
}

\author{
B. K. JENNINGS and R. K. BHADURI \\ Physics Department, McMaster University, Hamilton, Ontario, Canada \\ and \\ M. BRACK
}

Niels Bohr Institute, Copenhagen, Denmark

Received 30 June 1975

\begin{abstract}
For a system of noninteracting fermions in a one-body potential including spin-orbit interaction, an explicit series in the expansion parameter $\hbar^{2}$ is derived for the "smooth" part of the energy. The formalism is only valid for potentials with no discontinuous edges, and is based on the WignerKirkwood semiclassical partition function. Some numerical calculations are done in realistic spherical and deformed potentials to demonstrate the practical utility of the method. Numerical comparisons with the corresponding Strutinsky calculations are also made.
\end{abstract}

\section{Introduction}

For a given shell-model potential, one can define a quantity $E$ which is the sum of the occupied single particle energies of a $N$-nucleon system. This "energy" $E$, because of the shell structure of the orbitals, does not vary smoothly as a function of the nucleon number or the deformation parameters of the potential. By extracting from this system a smooth energy $\tilde{E}$ in a systematic manner, one can calculate the shell correction $\delta E=E-\tilde{E}$, and add this to the liquid-drop-model expression to obtain an accurate expression for the energy of the nucleus ${ }^{1}$ ).

The procedure generally employed to obtain $\widetilde{E}$ from a given set of single particle orbitals is due to Strutinsky ${ }^{1,2}$ ), and this amounts to a literal smoothing of the single particle level density by an appropriate choice of a smoothing function. In this paper we develop an alternative method for calculation of this smooth energy $\widetilde{E}$ which emphasizes the semiclassical nature of this quantity. In particular, for a local onebody potential that has no discontinuous edges, we derive an accurate and readily calculable expression for $\widetilde{E}$ in a power series of $\hbar$, involving the potential and its derivatives. The method is applicable for both spherical and deformed potentials, including a spin-orbit force of realistic strength. The furinulae that are derived are equally valid for infinite and finite potentials, and make no reference to the states in the continuum.

Since the Strutinsky method of obtaining $\widetilde{E}$ is an established procedure in wide use, it may be questioned why we are presenting an alternative method. In reply we may state that the method presented here is of interest both theoretically and 
in terms of practical calculations. Theoretically, our method of obtaining $\widetilde{E}$ amounts to doing an extended Thomas-Fermi calculation. The Thomas-Fermi term and the corrections to it are obtained in a systematic manner through the Wigner-Kirkwood expansion ${ }^{3,4}$ ) of the one-body partition function, with suitable modifications for the inclusion of the spin-orbit potential. In practice, we believe our method of calculating $\widetilde{E}$ is more accurate than the Strutinsky procedure for finite potentials. As is well known ${ }^{5,6}$ ), in the latter case smoothing involves a set of suitably chosen artificial discrete states in the continuum, obtained by diagonalizing the one-body Hamiltonian in a basis of optimum size. It is not generally realized, however, that to obtain an $\widetilde{E}$ independent of the Strutinsky smearing parameter, one has to apply the stationary condition ${ }^{6}$ ) in a delicate manner ${ }^{7,8}$ ), varying the curvature order to as high as sixteen. In our present method, on the other hand, the expression for $\widetilde{E}$ has no reference to the continuum and involves no free parameters. We also demonstrate that for realistic potential shapes the series obtained in the expansion parameter $\hbar$ is rapidly converging and that the first few terms are adequate to reproduce the desired accuracy in $\widetilde{E}$.

A number of papers ${ }^{8-11}$ ) have been published on the semiclassical method relating to the present problem, but the emphasis in these was to check the Strutinsky procedure in simple model cases. The objective of the present work is to develop the formalism for the calculation of $\tilde{E}$ in a realistic potential with spin-orbit force, and demonstrate the feasibility of the method by some numerical calculations. The formalism is developed in sect. 2, where a general expression for $\widetilde{E}$ is derived. In sect. 3 , this expression, which involves only the potential and its gradients, is evaluated for the model of a harmonic oscillator with constant spin-orbit strength, and the result checked directly by the evaluation of the partition function from the single particle spectrum. In sect. 4, numerical calculations for $\widetilde{E}$ are done for neutrons moving in Woods-Saxon type potentials, spherical or deformed, including realistic spin-orbit strengths. Comparison with the corresponding Strutinsky calculations are also made. Some of the more lengthy formulae are given in the appendix.

\section{The semiclassical approximation}

We shall first outline the derivation for a one-body potential $U(\boldsymbol{r})$ without the spin-orbit interaction $V_{L S}$, and then generalize it to include the effect of $V_{L S}$ in a perturbative approach. Let the one-body Hamiltonian operator be denoted by $\hat{H}_{0}=-\left(\hbar^{2} / 2 M\right) \nabla^{2}+U(\boldsymbol{r})$. The corresponding classical Hamiltonian is $H_{\mathrm{c}}=$ $p^{2} / 2 M+U(\boldsymbol{r})$. The quantum partition function is given by

$$
Z^{0}(\beta)=\operatorname{tr} \mathrm{e}^{-\beta \hat{\mathbf{H}}_{0}}
$$


while the classical one is

$$
Z_{\mathrm{c}}^{O}(\beta)=\frac{2}{h^{3}} \int \mathrm{e}^{-\beta H_{c}} \mathrm{~d}^{3} p \mathrm{~d}^{3} r
$$

where the factor of two comes from spin degeneracy. The integration over $p$ in the above equation can be done analytically to yield

$$
Z_{\mathrm{c}}^{0}(\beta)=\frac{1}{4 \pi^{\frac{3}{2}} \beta^{\frac{3}{2}}}\left(\frac{2 M}{\hbar^{2}}\right)^{\frac{3}{2}} \int \mathrm{e}^{-\beta U(\boldsymbol{r})} \mathrm{d}^{3} r .
$$

Note that $Z^{0}(\beta)=\mathscr{L}_{\beta} g(\varepsilon)$, where $g(\varepsilon)=\sum_{i} \delta\left(\varepsilon-\varepsilon_{i}\right)$ is the single particle density of states, and $\mathscr{L}_{\beta}$ denotes the Laplace transform with respect to $\beta$. The simplest way to wash out quantum shell effects would be to calculate the energy of the system using the classical density of states $g_{\mathrm{c}}(\varepsilon)$, obtained through Laplace inversion of the classical partition function: $g_{\mathrm{c}}(\varepsilon)=\mathscr{L}_{\varepsilon}^{-1} Z_{\mathrm{c}}^{0}(\beta)$. For a given number of neutrons $N$, the Fermi energy $\lambda_{\mathrm{c}}$ in this case is determined by

$$
N=\int_{0}^{\lambda_{c}} g_{\mathrm{c}}(\varepsilon) \mathrm{d} \varepsilon=\mathscr{L}_{\lambda_{\mathrm{c}}}^{-1}\left(\frac{Z_{\mathrm{c}}^{0}(\beta)}{\beta}\right)
$$

and the energy of the system is

$$
\widetilde{E}_{\mathrm{c}}=\int_{0}^{\lambda_{\mathrm{c}}} \varepsilon g_{\mathrm{c}}(\varepsilon) \mathrm{d} \varepsilon=N \lambda_{\mathrm{c}}-\mathscr{L}_{\lambda_{\mathrm{c}}}^{-1}\left(\frac{Z_{\mathrm{c}}^{0}(\beta)}{\beta^{2}}\right) .
$$

Making use of eqs. (3)-(5), and noting that

$$
\mathscr{L}_{\lambda_{\mathrm{c}}}^{-1} \frac{1}{\beta^{n}} \mathrm{e}^{-\beta U}=\frac{\left(\lambda_{\mathrm{c}}-U\right)^{n-1}}{\Gamma(n)} \theta\left(\lambda_{\mathrm{c}}-U\right),
$$

where $\Gamma(n)$ is the usual gamma function and $\theta\left(\lambda_{\mathrm{c}}-U\right)$ is : e unit step function, it immediately follows that

$$
\begin{gathered}
N=\frac{1}{\left(3 \pi^{2}\right)}\left(\frac{2 M}{\hbar^{2}}\right)^{\frac{3}{2}} \int^{r_{\mathrm{c}}} \mathrm{d}^{3} r\left(\lambda_{\mathrm{c}}-U\right)^{\frac{3}{2}}, \\
\widetilde{E}_{\mathrm{c}}=\frac{1}{\left(3 \pi^{2}\right)}\left(\frac{2 M}{\hbar^{2}}\right)^{\frac{3}{2}} \int^{r_{\mathrm{c}}} \mathrm{d}^{3} r\left[\frac{3}{5}\left(\lambda_{\mathrm{c}}-U\right)^{\frac{5}{2}}+U\left(\lambda_{\mathrm{c}}-U\right)^{\frac{3}{2}}\right] .
\end{gathered}
$$

Here the integrals are cut off at the classical turning point $\boldsymbol{r}_{\mathrm{c}}$ such that $U\left(\boldsymbol{r}_{\mathrm{c}}\right)=\lambda_{\mathrm{c}}$. The expressions (7) and (8) are just the standard Thomas-Fermi (TF) equations, which thus arise from the classical density of states. It follows that one may obtain correction terms to the TF result by making systematic corrections to the classical partition function $Z_{\mathrm{c}}^{\mathrm{o}}(\beta)$. Just such a semiclassical partition function, $Z_{\mathrm{sc}}^{\mathrm{o}}(\beta)$, was developed by Wigner ${ }^{3}$ ) and Kirkwood ${ }^{4}$ ), in which the correction terms appear as a power series in $\hbar$. Note that by taking a plane-wave basis, one formally expresses eq. (1) for the quantum partition function as 


$$
Z^{0}(\beta)=\frac{2}{h^{3}} \int \mathrm{e}^{-i p \cdot r / \hbar} \mathrm{e}^{-\beta \hat{H}_{0}} \mathrm{e}^{i p \cdot r / \hbar} \mathrm{d}^{3} p \mathrm{~d}^{3} r
$$

In order to get the semiclassical result, one writes

$$
\mathrm{e}^{-\beta \hat{H}_{0}} \mathrm{e}^{i \boldsymbol{p} \cdot \boldsymbol{r} / \hbar}=\mathrm{e}^{-\boldsymbol{\beta} \boldsymbol{H}_{\mathrm{c}}} \mathrm{e}^{i \boldsymbol{p} \cdot \boldsymbol{r} / \boldsymbol{\hbar}} w(\boldsymbol{r}, \boldsymbol{p}, \boldsymbol{\beta}) \equiv u(\boldsymbol{r}, \boldsymbol{p}, \boldsymbol{\beta}),
$$

so that $u$ satisfies the Bloch equation

$$
\frac{\partial u}{\partial \beta}+\hat{H}_{0} u=0
$$

with the boundary condition $\lim _{\beta \rightarrow 0} u=\exp (i p \cdot r / \hbar)$. The Bloch equation is rewritten in terms of $w$ by using eq. (10). It is then solved to each order in $\hbar$ by writing

$$
w=1+\hbar w_{1}+\hbar^{2} w_{2}+\ldots
$$

and obtaining explicit expressions for $w_{1}, w_{2}$, etc. in terms of the classical momentum vector $\boldsymbol{p}$ and the gradients of the potential $U(\boldsymbol{r})$. Finally, using eqs. (9)-(11), the semiclassical partition function $Z_{\mathrm{sc}}^{0}(\beta)$ is written as

$$
Z_{\mathrm{sc}}^{0}(\beta)=\frac{2}{h^{3}} \int \mathrm{e}^{-\beta H_{\mathrm{c}}}\left(1+\hbar w_{1}+\hbar^{2} w_{2}+\ldots\right) \mathrm{d}^{3} p \mathrm{~d}^{3} r
$$

The expression for $w_{1}$ and $w_{2}$ may be found in ref. ${ }^{12}$ ). Note that the method is only applicable for potentials whose first and higher derivatives exist. On doing the $p$ integrations in (12), $w_{1}, w_{3}$, etc, which are imaginary and have odd powers of $p$, vanish. Keeping terms up to $w_{4}$, we get ${ }^{\dagger}$

$$
\begin{aligned}
Z_{\mathrm{sc}}^{0}(\beta)=\frac{1}{4 \pi^{\frac{3}{2} \beta^{\frac{3}{2}}}\left(\frac{2 M}{\hbar^{2}}\right)^{\frac{3}{2}} \int} & \mathrm{d}^{3} r \mathrm{e}^{-\beta U}\left[1-\frac{\beta^{2}}{12} \frac{\hbar^{2}}{2 M} \nabla^{2} U\right. \\
& \left.+\frac{\beta^{3}}{1440}\left(\frac{\hbar^{2}}{2 M}\right)^{2}\left(-7 \nabla^{4} U+5 \beta\left(\nabla^{2} U\right)^{2}+\beta \nabla^{2}(\nabla U)^{2}\right)\right]
\end{aligned}
$$

where the first term is just the classical term (3), while the second and third terms arise from $w_{2}$ and $w_{4}$ respectively. The expressions for $N$ and $\tilde{E}_{\text {sc }}$, using eqs. (4) and (5) where $Z_{\mathrm{c}}^{0}$ has been replaced by $Z_{\mathrm{sc}}^{0}$, can be obtained in a straightforward manner. The complete expressions for $N$ and $\tilde{E}_{\mathrm{sc}}$ will be given later after we show how to include spin-orbit effects in the semiclassical partition function (13).

We now include the spin-orbit potential and the one-body Hamiltonian becomes

$$
\hat{H}=\hat{H}_{0}+\widehat{V}_{L S},
$$

'In eq. (13) of ref. ${ }^{12}$ ), the coefficient of $\nabla U \cdot \nabla \nabla^{2} U$ should be 8 and not 4 , but their final expressions for the virial coefficients are correct. 
where $\hat{H}_{0}$ has been defined earlier, and

$$
\begin{aligned}
\hat{V}_{L S} & =-\frac{\kappa}{M}(\nabla f \times \hat{\boldsymbol{p}}) \cdot \hat{\mathbf{s}} \\
& =i \kappa \frac{\hbar^{2}}{2 M}(\nabla f \times \nabla) \cdot \hat{\boldsymbol{\sigma}} .
\end{aligned}
$$

Here $\kappa$ is a dimensionless strength factor, $f(\boldsymbol{r})$ is a dimensionless form factor and we have used the retations $\hat{\boldsymbol{p}}=-i \hbar \nabla$ and $\hat{\boldsymbol{s}}=\frac{1}{2} \hbar \hat{\boldsymbol{\sigma}}$, where $\hat{\boldsymbol{\sigma}}$ is the unit Pauli matrix. Now the quantum partition function becomes:

$$
Z(\beta)=\operatorname{tr} \mathrm{e}^{-\beta\left(\hat{H}_{0}+\hat{V}_{L S}\right)} .
$$

Since $\hat{V}_{L S}$ in general does not commute with $\hat{H}_{0}$, we cannot write $Z(\beta)$ as the trace over a product of two exponentials. However, by writing

$$
\mathrm{e}^{-\beta\left(\hat{H}_{0}+\hat{V}_{L S}\right)}=\mathrm{e}^{-\beta \hat{H}_{0}} \hat{S}(\beta),
$$

we can obtain a perturbative expansion, in powers of $V_{L S}(\beta)$ :

$$
\hat{S}(\beta)=1-\int_{0}^{\beta} \hat{V}_{L S}\left(\beta^{\prime}\right) \mathrm{d} \beta^{\prime}+\int_{0}^{\beta} \hat{V}_{L S}\left(\beta^{\prime}\right) \int_{0}^{\beta^{\prime}} \hat{V}_{L S}\left(\beta^{\prime \prime}\right) \mathrm{d} \beta^{\prime \prime} \mathrm{d} \beta^{\prime}-\ldots
$$

where

$$
\hat{V}_{L S}(\beta)=\mathrm{e}^{\beta \hat{H}_{0}} \hat{V}_{L S} \mathrm{e}^{-\beta \hat{H}_{0}}=\hat{V}_{L S}+\beta\left[\hat{H}_{0}, \hat{V}_{L S}\right]+\frac{\beta^{2}}{2 !}\left[\hat{H}_{0},\left[\hat{H}_{0}, \hat{V}_{L S}\right]\right]+\ldots
$$

From (16) and (17), using the plane-wave basis, we get

$$
\begin{aligned}
Z(\beta)=\frac{1}{h^{3}} \sum_{\sigma} \int_{\mathrm{e}^{-i \boldsymbol{p} \cdot \boldsymbol{r} / \hbar} \mathrm{e}^{-\beta \hat{H}_{0}} \hat{S}(\beta) \mathrm{e}^{i \boldsymbol{p} \cdot \boldsymbol{r} / h} \mathrm{~d}^{3} p \mathrm{~d}^{3} r} \\
=\frac{1}{h^{3}} \sum_{\sigma} \int\left(\mathrm{e}^{-\beta \hat{H}_{0}} \mathrm{e}^{i \boldsymbol{p} \cdot \boldsymbol{r} / \hbar}\right) * \hat{S}(\beta) \mathrm{e}^{i \boldsymbol{p} \cdot \boldsymbol{r} / \hbar} \mathrm{d}^{3} p \mathrm{~d}^{3} r .
\end{aligned}
$$

We can now use the semiclassical eqs. (10) and (11), and further put

$$
\hat{S}(\beta) \mathrm{e}^{i \boldsymbol{p} \cdot \boldsymbol{r} / \hbar}=\mathrm{e}^{i \boldsymbol{p} \cdot \boldsymbol{r} / \hbar} I(\boldsymbol{r}, \boldsymbol{p}, \beta),
$$

to obtain

$$
Z_{\mathrm{sc}}(\beta)=\frac{1}{h^{3}} \sum_{\sigma} \int \mathrm{e}^{-\beta H_{c}}\left(1+\hbar w_{1}^{*}+\hbar^{2} w_{2}+\ldots\right) I(\boldsymbol{r}, \boldsymbol{p}, \beta) \mathrm{d}^{3} r \mathrm{~d}^{3} p
$$

The formal derivation of the semiclassical partition function with the inclusion of the spin-orbit potential is now complete, and a great deal of algebra is necessary to evaluate it explicitly to any desired order in powers of $\kappa$. The final result may be written as

$$
Z_{\mathrm{sc}}(\beta)=Z_{\mathrm{sc}}^{0}(\beta)+Z_{L \mathrm{~s}}(\beta)
$$


where $Z_{\mathrm{se}}^{0}(\beta)$ is defined by eq. (13), and $Z_{L S}(\beta)$ is given by

$$
\begin{aligned}
Z_{L S}(\beta) & =\frac{\kappa^{2}}{8 \pi^{\frac{3}{2}}} \frac{1}{\beta^{\frac{1}{2}}}\left(\frac{2 M}{\hbar^{2}}\right)^{\frac{1}{2}} \int \mathrm{d}^{3} r \mathrm{e}^{-\beta U}(\nabla f)^{2} \\
+ & \frac{1}{96 \pi^{\frac{3}{2}}} \beta^{\frac{1}{2}}\left(\frac{\hbar^{2}}{2 M}\right)^{\frac{1}{2}} \int \mathrm{d}^{3} r \mathrm{e}^{-\beta U}\left[\kappa ^ { 2 } \left\{-\beta(\nabla f)^{2}\left(\nabla^{2} U\right)\right.\right. \\
& \left.+\frac{1}{2} \nabla^{2}(\nabla f)^{2}-\left(\nabla^{2} f\right)^{2}+\nabla f \cdot \nabla\left(\nabla^{2} f\right)\right\}-2 \kappa^{3}\left\{(\nabla f)^{2} \nabla^{2} f-\frac{1}{2} \nabla f \cdot \nabla(\nabla f)^{2}\right\} \\
& \left.+2 \kappa^{4}(\nabla f)^{4}\right],
\end{aligned}
$$

where the first term is proportional to $\hbar^{-1}$, and is of the same order as the contribution $w_{2}$ to $Z_{\mathrm{sc}}^{0}$ (see eq. (13)), while the next term is smaller by a factor of $\hbar^{2}$. In $Z_{\mathrm{sc}}(\beta)$ of eq. (22), we have therefore included all terms of order $\hbar^{-3}, \hbar^{-1}$ and $\hbar$ with powers in $\kappa$ up to four. The convergence in the resulting series of $\tilde{E}_{\mathrm{sc}}$ will be discussed in the next two sections when the harmonic oscillator and the Woods-Saxon type potentials with realistic values of $\kappa$ are taken. The terms in $Z_{L S}$ of even powers in $\kappa$ and of leading order in $\hbar$, i.e. of order $\hbar^{-1}$ in $\kappa^{2}$, of $\hbar$ in $\kappa^{4}$, etc. may also be derived in a simple but nonrigorous manner. Taking the Hamiltonian given by eqs. (14) and (15), and neglecting the corrections of higher order in $\hbar$ that arise due to the noncommutation of $\hat{H}_{0}$ and $\hat{V}_{L S}$, the "classical" partition function may be written as

$$
Z_{\mathrm{c}}(\beta)=\frac{1}{h^{3}} \int \mathrm{e}^{-\left(p^{2} / 2 M+U\right) \beta} \sum_{\sigma} \exp [\beta(\kappa \hbar / 2 M)|\nabla f \times p| \cos \theta] \mathrm{d}^{3} p \mathrm{~d}^{3} r,
$$

where $\cos \theta= \pm 1$ for the two possible spin orientations.

Therefore

$$
Z_{\mathrm{c}}(\beta)=\frac{2}{h^{3}} \int \mathrm{e}^{-\left(p^{2} / 2 M+U\right) \beta} \cosh \left(\frac{\beta \kappa \hbar}{2 M}|\nabla f \times p|\right) \mathrm{d}^{3} p \mathrm{~d}^{3} r .
$$

Since $\cosh x=1+\frac{1}{2} x^{2}+\ldots$, we see that the first term yields just the classical $Z_{\mathrm{c}}^{0}$, while the leading term involving $V_{L S}$ is

$$
Z_{L S}(\beta) \approx \frac{1}{\hbar} \frac{\beta^{2} \kappa^{2}}{32 \pi^{3} M^{2}} \int(\nabla f \times p)^{2} \mathrm{e}^{-\left(p^{2} / 2 M+U\right) \beta} \mathrm{d}^{3} p \mathrm{~d}^{3} r .
$$

Since the integral

$$
\int(\nabla f \times p)^{2} \mathrm{e}^{-\beta\left(p^{2} / 2 M\right)} \mathrm{d}^{3} p=\left(\frac{2 M}{\beta}\right)^{\frac{3}{2}} \pi^{\frac{3}{2}}(\nabla f)^{2},
$$

we get exactly the first term of eq. (23).

Our objective is to find an expression for the semiclassical energy $\widetilde{E}_{\mathrm{sc}}$, which not only has the leading classical term of order $\hbar^{-3}$ given by eq. (8), but also correction terms of order $\hbar^{-1}$ and $\hbar$. The semiclassical $Z_{\mathrm{sc}}$ that we have derived may now be used in eq. (4) to obtain the Fermi energy $\lambda_{\text {sc }}$, and then in eq. (5) to find $\widetilde{E}_{\text {sc }}$. It is shown in appendix A, however, that $Z_{\mathrm{sc}}$ need only be taken to order $\hbar^{-1}$ in eq. (4) to find $\lambda_{\text {sc }}$, but terms of order $\hbar$ in $Z_{\text {sc }}$ should be included in eq. (5) to obtain $\widetilde{E}_{\text {sc }}$ 
correct to order $\hbar$. Following this procedure, we obtain

$$
\begin{aligned}
N=\frac{1}{3 \pi^{2}}\left(\frac{2 M}{\hbar^{2}}\right)^{\frac{3}{2}} \int^{r_{\mathrm{sc}}} \mathrm{d}^{3} r\left[\left(\lambda_{\mathrm{sc}}-U\right)^{\frac{3}{2}}+\frac{\hbar^{2}}{2 M}\left(\frac{3}{4} \kappa^{2}(\nabla f)^{2}\left(\lambda_{\mathrm{sc}}-U\right)^{\frac{1}{2}}\right.\right. & \left.\left.-\frac{1}{16} \nabla^{2} U\left(\lambda_{\mathrm{sc}}-U\right)^{-\frac{1}{2}}\right)\right],
\end{aligned}
$$

where the turning point $\boldsymbol{r}_{\mathrm{sc}}$ is defined by the relation $U\left(\boldsymbol{r}_{\mathrm{sc}}\right)=\lambda_{\mathrm{sc}}$. In the expression for $\widetilde{E}_{\mathrm{sc}}$, in order to get inverse Laplace transforms in convergent forms, the following identity is exploited:

$$
\mathrm{e}^{-\beta U}=-\frac{1}{\beta} \frac{\nabla U \cdot \nabla \mathrm{e}^{-\beta U}}{(\nabla U)^{2}}
$$

The resulting expression for $\widetilde{E}_{\mathrm{sc}}$ is given by

$$
\begin{aligned}
\tilde{E}_{\mathrm{sc}} & =\lambda_{\mathrm{sc}} N-\frac{2}{15 \pi^{2}}\left(\frac{2 M}{\hbar^{2}}\right)^{\frac{3}{2}} \int \mathrm{d}^{3} r\left(\lambda_{\mathrm{sc}}-U\right)^{\frac{3}{2}} \\
& +\frac{1}{24 \pi^{2}}\left(\frac{2 M}{\hbar^{2}}\right)^{\frac{1}{2}} \int \mathrm{d}^{3} r\left(\lambda_{\mathrm{sc}}-U\right)^{\frac{1}{2}}\left[\nabla^{2} U-4 \kappa^{2}(\nabla f)^{2}\left(\lambda_{\mathrm{sc}}-U\right)\right] \\
& -\frac{1}{5760 \pi^{2}}\left(\frac{\hbar^{2}}{2 M}\right)^{\frac{1}{2}} \int \mathrm{d}^{3} r \frac{1}{\left(\lambda_{\mathrm{sc}}-U\right)^{\frac{1}{2}}(\nabla U)^{2}}\left[-7 \nabla^{4} U(\nabla U)^{2}+5\left(\nabla^{2} U\right)^{3}\right. \\
& +10\left[\nabla U \cdot \nabla\left(\nabla^{2} U\right)\right] \nabla^{2} U-5\left(\nabla^{2} U\right)^{2} \nabla U \cdot \nabla(\nabla U)^{2} /(\nabla U)^{2} \\
& \left.+\left(\nabla^{2} U\right) \nabla^{2}(\nabla U)^{2}+\nabla U \cdot \nabla \nabla^{2}(\nabla U)^{2}-\nabla^{2}(\nabla U)^{2} \nabla U \cdot \nabla(\nabla U)^{2} /(\nabla U)^{2}\right] \\
& -\frac{1}{48 \pi^{2}}\left(\frac{\hbar^{2}}{2 M}\right)^{\frac{1}{2}} \int \mathrm{d}^{3} r\left(\lambda_{\mathrm{sc}}-U\right)^{\frac{1}{2}}\left[\kappa ^ { 2 } \left\{\frac{1}{2} \nabla^{2}(\nabla f)^{2}-\left(\nabla^{2} f\right)^{2}\right.\right. \\
& \left.+\nabla f \cdot \nabla\left(\nabla^{2} f\right)-\frac{(\nabla f)^{2} \nabla^{2} U}{2\left(\lambda_{\mathrm{sc}}-U\right)}\right\}-2 \kappa^{3}\left\{(\nabla f)^{2} \nabla^{2} f-\frac{1}{2} \nabla f \cdot \nabla(\nabla f)^{2}\right\} \\
& \left.+2 \kappa^{4}(\nabla f)^{4}\right],
\end{aligned}
$$

where all the integrals are cut off at the turning point $\boldsymbol{r}_{\mathrm{sc}}$, as defined earlier. Although this equation looks formidable, it is straightforward to evaluate numerically when $U(\boldsymbol{r})$ and $f(\boldsymbol{r})$ are given analytically and their derivatives are finite. For spherically symmetric $U$ and $f$, eq. (25) is considerably simplified, and the resulting expression for $\widetilde{E}_{\mathrm{sc}}$ is given in appendix B. For the axially symmetric deformed case, it is best to use cylindrical coordinates to evaluate the gradient terms, and the integrals involved are two-dimensional. Results of numerical calculations using eqs. (24) and (25) are reported in sect. 4. 


\section{An illustrative example}

In this section, we consider a simple model where the one-body potential is an isotropic harmonic oscillator with a constant spin-orbit interaction. The object of this exercise is two-fold. In this case $Z_{\mathrm{sc}}$ can be directly obtained from the eigen energy spectrum without going through the Wigner-Kirkwood expansion. The relevant equations for $Z_{\text {sc }}$ and $\widetilde{E}_{\mathrm{sc}}$ derived in the earlier section can therefore be checked here. Also, for reasonable values of the oscillator parameter and the spinorbit strength $\kappa$, the convergence of the series can be examined.

The one-body Hamiltonian is taken to be

$$
\hat{H}=-\frac{\hbar^{2}}{2 M} \nabla^{2}+\frac{1}{2} M \omega^{2} r^{2}-\kappa(\hbar \omega) \hat{\boldsymbol{l}} \cdot \hat{\boldsymbol{\sigma}}
$$

where $\hat{l}=-i(\boldsymbol{r} \times \nabla)$, and $\hat{\boldsymbol{\sigma}}$ is the unit Pauli matrix. Comparing $\hat{V}_{L S}$ of the above Hamiltonian with eq. (15), we find that for this case the form factor $f$ is simply

$$
f(r)=(M \omega / \hbar) r^{2}
$$

The resulting single particle spectrum is given by

$$
\begin{gathered}
\varepsilon_{j=l+\frac{1}{2}}=\left(2 n+l+\frac{3}{2}\right) \hbar \omega-\kappa \hbar \omega l, \\
\varepsilon_{j=l-\frac{1}{2}}=\left(2 n+l+\frac{3}{2}\right) \hbar \omega+\kappa \hbar \omega(l+1) .
\end{gathered}
$$

The degeneracy of the states for a given $j$ is $(2 j+1)$; for the $j=l+\frac{1}{2}$ states this is $2(l+1)$, and for the $j=l-\frac{1}{2}$ states this is $2 l$. The quantum partition function is

$$
Z(\beta)=2 \mathrm{e}^{-\frac{3}{2} \hbar \omega \beta} \sum_{n=0}^{\infty} \mathrm{e}^{-2 n \hbar \omega \beta} \sum_{l=0}^{\infty}\left[(l+1) \mathrm{e}^{-\hbar \omega \beta l(1-\kappa)}+l \mathrm{e}^{-\hbar \omega \beta l(1+\kappa)} \mathrm{e}^{-\beta \hbar \omega \kappa}\right] .
$$

The sums over $n$ and $l$ can be done explicitly to give

$$
\begin{aligned}
Z(\beta)=\frac{\mathrm{e}^{-\frac{1}{2} \hbar \omega \beta}}{4 \sinh (\hbar \omega \beta)}\left[\frac{1}{\sinh ^{2}\left[\frac{1}{2} \hbar \omega \beta(1-\kappa)\right]}+\frac{\mathrm{e}^{-\kappa \hbar \omega \beta}}{\sinh ^{2}\left[\frac{1}{2} \hbar \omega \beta(1+\kappa)\right]}\right. \\
\left.+\frac{2 \mathrm{e}^{\frac{1}{2} \hbar \omega \beta(1-\kappa)}}{\sinh \left[\frac{1}{2} \hbar \omega \beta(1-\kappa)\right]}\right] .
\end{aligned}
$$

Note that for $\kappa=0$, the right hand side of the above equation reduces to the well known form $\frac{1}{4} \operatorname{cosech}^{3}\left(\frac{1}{2} \hbar \omega \beta\right)$. Expanding the hyperbolic and exponential functions 
in powers of $\hbar$, and collecting terms of the same order in $\hbar$, we get the semiclassical partition function

$$
\begin{aligned}
Z_{\mathrm{sc}}(\beta)=\frac{1}{\left(1-\kappa^{2}\right)^{2}} & {\left[\frac{2}{(\hbar \omega \beta)^{3}}\left(1+\kappa^{2}\right)-\frac{2 \kappa^{3}}{(\hbar \omega \beta)^{2}}-\frac{1}{12 \hbar \omega \beta}\left(3+9 \kappa^{2}-10 \kappa^{4}\right)\right.} \\
+ & \left.\frac{1}{12}\left(5 \kappa^{3}-2 \kappa^{5}\right)+\frac{\hbar \omega \beta}{2880}\left(51+303 \kappa^{2}-364 \kappa^{4}+24 \kappa^{6}\right)+\ldots\right]
\end{aligned}
$$

In the above equation for $Z_{\mathrm{sc}}$ we have obtained an expansion in powers of $\hbar$, whereas the Wigner-Kirkwood series, given by eqs. (13), (22) and (23), the successive terms differed by $\hbar^{2}$. This apparent contradiction is easily resolved when we note that in the present model the form factor $f(r)$, as given by eq. (27) contains an explicit factor of $\hbar^{-1}$. This will not be so when more realistic forms for $f(r)$ are chosen in the next section.

For the Hamiltonian (26), a reasonable value for $\kappa$ is $\approx 0.1$, so that we may further expand $\left(1-\kappa^{2}\right)^{-2}$ in eq. (30). It is then convenient to split the resulting $Z_{\mathrm{sc}}$ as in eq. (22), writing

$$
Z_{\mathrm{sc}}^{0}=Z_{\mathrm{sc}}^{0}+Z_{L S}
$$

We then get

$$
Z_{\mathrm{sc}}^{0}=\frac{2}{(\hbar \omega \beta)^{3}}-\frac{1}{4(\hbar \omega \beta)}+\frac{17}{960} \hbar \omega \beta-\ldots
$$

and $Z_{L S}$ consists of the rest of the terms in (30) involving the powers of $\kappa$. It is easy to verify that eq. (13), which makes no use of the eigenspectrum, exactly reproduces eq. (31) when we put $U=\frac{1}{2} M \omega^{2} r^{2}$ and perform the relevant integrations. When writing $Z_{L S}$, note that two small expansion parameters are involved, $\hbar$ and $\kappa$. Arranging the terms in the expansion such that all terms within a square bracket are of the same order, we may write

$$
\begin{aligned}
Z_{L S}= & {\left[\frac{6 \kappa^{2}}{(\hbar \omega \beta)^{3}}\right]+\left[\frac{10 \kappa^{4}}{(\hbar \omega \beta)^{3}}-\frac{2 \kappa^{3}}{(\hbar \omega \beta)^{2}}-\frac{5}{4} \frac{\kappa^{2}}{\hbar \omega \beta}\right] } \\
& +\left[\frac{14 \kappa^{6}}{(\hbar \omega \beta)^{3}}-\frac{4 \kappa^{5}}{(\hbar \omega \beta)^{2}}-\frac{17}{12} \frac{\kappa^{4}}{\hbar \omega \beta}+\frac{5}{12} \kappa^{3}+\frac{9}{64} \kappa^{2}(\hbar \omega \beta)\right]+\ldots
\end{aligned}
$$

It is again easy to check that all the terms in the first two brackets in the above equation are reproduced by the Wigner-Kirkwood formula (23) by putting $f=$ $(M \omega / \hbar) r^{2}$ there. We shall presently show that the terms in the last bracket of eq. (32) contribute insignificantly to $\widetilde{E}_{\mathrm{sc}}$.

For a given number of particles, $\lambda_{\mathrm{sc}}$ can now be calculated using $Z_{\mathrm{sc}}$ of eqs. (31) and (32). As explained earlier and in appendix $A$, it is enough in this calculation to 
take for $Z_{\text {sc }}$ only the first two terms of eq. (31) and the first term of (32). The smooth energy $\widetilde{E}_{\mathrm{sc}}$ is then calculated with this $\lambda_{\mathrm{sc}}$ using the formula

$$
\tilde{E}_{\mathrm{sc}}=\lambda_{\mathrm{sc}} N-\mathscr{L}_{\lambda_{\mathrm{sc}}}^{-1}\left[Z_{\mathrm{sc}}(\beta) / \beta^{2}\right],
$$

with all terms in $Z_{\mathrm{sc}}$ now included. If we neglect the terms in the third bracket of eq. (32), the resulting expression for $\widetilde{E}_{\mathrm{sc}}$ is

$$
\begin{aligned}
\left(\tilde{E}_{\mathrm{sc}}-\lambda_{\mathrm{sc}} N\right)=-\frac{\lambda_{\mathrm{sc}}^{4}}{12(\hbar \omega)^{3}}+\frac{\lambda_{\mathrm{sc}}^{2}}{8 \hbar \omega}-\frac{17}{960} \hbar \omega & \\
& \quad-\frac{\kappa^{2}}{4} \frac{\lambda_{\mathrm{sc}}^{4}}{(\hbar \omega)^{3}}+\left(\frac{5}{8} \kappa^{2} \frac{\lambda_{\mathrm{sc}}^{2}}{\hbar \omega}+\frac{\kappa^{3}}{3} \frac{\lambda_{\mathrm{sc}}^{3}}{(\hbar \omega)^{2}}-\frac{5}{12} \kappa^{4} \frac{\lambda_{\mathrm{sc}}^{4}}{(\hbar \omega)^{3}}\right) .
\end{aligned}
$$

It is not difficult to check that the above equation is also reproduced if one uses the Wigner-Kirkwood form (25) and substitutes $U=\frac{1}{2} M \omega^{2} r^{2}$ and $f=(M \omega / \hbar) r^{2}$. In the above expression, the first, second and third terms on the r.h.s. arise from the corresponding terms of eq. (31) for $Z_{\mathrm{sc}}^{0}$, the fourth term comes from the first term of eq. (32) for $Z_{L S}$ and the last three terms of eq. (34) arise from the second bracket of eq. (32). To estimate the relative importance of these terms in a heavy nucleus, we assume $\hbar \omega=7 \mathrm{MeV}$ and arbitrarily set $\lambda_{\mathrm{sc}}=42 \mathrm{MeV}$. The first three terms of eq. (34) then are $-756,31.5$ and $-0.12 \mathrm{MeV}$; the leading contribution of $Z_{L S}$ is given by the fourth term and is $-22.68 \mathrm{MeV}$. The last three terms of eq. (34) give 1.70 $\mathrm{MeV}$. If we had included the third bracket in eq. (32) for $Z_{L S}$, it would contribute only $-0.005 \mathrm{MeV}$ to $\widetilde{E}_{\mathrm{sc}}-\lambda_{\mathrm{sc}} N$. In the second bracket of eq. (32), it is the $\kappa^{2}$ term that contributes most; 1.58 out of $1.70 \mathrm{MeV}$. This happens since the $\kappa^{3}$ and $\kappa^{4}$ terms in this bracket, each of the order of $0.5 \mathrm{MeV}$, nearly cancel each other.

We conclude from all this that the convergence of the series (23), or equivalently, (34), is satisfactory to within $0.1 \mathrm{MeV}$. It is quite justifiable to neglect the last five terms of eq. (32) for $Z_{L S}$, which contribute only about $0.005 \mathrm{MeV}$ to $\widetilde{E}_{\mathrm{sc}}$ in this model.

\section{Numerical calculations}

In this section we shall demonstrate the practical usefulness of the formalism developed in sect. 2 by performing some numerical calculations for $\widetilde{E}_{\mathrm{sc}}$ in realistic spherical and deformed potential wells. Taking a few examples, we again focus our attention on the convergence of the semiclassical series, and we also compare our results with the corresponding numbers obtained through carefully done Strutinsky calculations. For these objectives, it is enough to consider only neutrons moving in some average potential.

In the first example, we consider $N$ neutrons to be moving in a spherical WoodsSaxon well, as parametrized by Blomquist and Wahlborn ${ }^{13}$ ). For this potential, unambiguous Strutinsky results are available ${ }^{14}$ ) for $N=126$ and 184 . The central 
part of the potential is given by ${ }^{14}$ )

$$
U(r)=-V_{0} f(r)
$$

and the spin-orbit part is, in the notation of eq. (15),

$$
V_{L S}=-\kappa \frac{\hbar^{2}}{2 M} \frac{1}{r} \frac{\mathrm{d} f}{\mathrm{~d} r} \hat{\boldsymbol{l}} \cdot \hat{\boldsymbol{\sigma}},
$$

where $\hat{l}=-i(r \times \nabla)$. The form for $f(r)$ is

$$
f(r)=[1+\exp (r-R) / a]^{-1} .
$$

The numerical values of the parameters for $N=126$ are $V_{0}=44 \mathrm{MeV}, R=7.52$ fm, $a=0.67 \mathrm{fm}$ and $\kappa=-0.7491$; and for $N=184$ these are $V_{0}=43 \mathrm{MeV}, R=8.48$ $\mathrm{fm}, a=0.67 \mathrm{fm}$ and $\kappa=-0.7321$. Compared to the harmonic oscillator model of sect. 3, the sign of $\kappa$ here is opposite, and its apparent magnitude much larger. This comes about because $(1 / r) \mathrm{d} f / \mathrm{d} r$ is negative here and it is nonzero only in the surface region, so that a larger $\kappa$ is needed to yield the same spin-orbit splitting. For the same reason, in spite of the apparent increase in $\kappa$, the contribution of $V_{L S}$ to $\tilde{E}_{\mathrm{sc}}$ is of the same order of magnitude as in the harmonic model, and the perturbation scheme remains satisfactory.

For a given $N$, the Fermi energy $\lambda_{\text {sc }}$ is first computed from eq. (24). In order to examine the convergence of the series for $\widetilde{E}_{\mathrm{sc}}$, we write eq. (33) as

$$
\widetilde{E}_{\mathrm{sc}}=\lambda_{\mathrm{sc}} N-\mathscr{L}^{-1}\left[Z_{\mathrm{sc}}^{0} / \beta^{2}\right]-\mathscr{L}^{-1}\left[Z_{L S} / \beta^{2}\right] .
$$

This equation reduces to the form (25) when terms of order $\hbar^{-3}, \hbar^{-1}$ and $\hbar$ are retained in $Z_{\mathrm{sc}}^{0}$, and terms of order $\hbar^{-1}$ and $\hbar$ are kept in $Z_{L S}$. Quite generally, then, we may write

$$
\widetilde{E}_{\mathrm{sc}}=\lambda_{\mathrm{sc}} N-\left[E_{-3}^{0}+E_{-1}^{0}+E_{1}^{0}\right]-\left[E_{-1}^{L S}+E_{1}^{L S}\right],
$$

where $E_{-n}^{0}$ is the contribution of order $\hbar^{-n}$ arising from $\mathscr{L}^{-1}\left[Z_{\mathrm{sc}}^{0} / \beta^{2}\right]$, and likewise $E_{-n}^{L S}$ is of order $\hbar^{-n}$ coming from $\mathscr{L}^{-1}\left[Z_{L S} / \beta^{2}\right]$ in (36). The analytical expression for the various terms of eq. (37) for the spherical case are given in eq. (B.2) of the appendix, and their numerical values for $N=126$ and $N=184$ are displayed in table 1 . Also given for comparison, in the third line of this table, are the corresponding numbers in the harmonic oscillator model that were obtained in sect. 3. Although the convergence of the series for the Woods-Saxon case is not as good as in the harmonic oscillator model, it is still adequate. This is so since we noted in the last section that the next higher order contribution of $V_{L S}$ to $\widetilde{E}_{\mathrm{sc}}$ in the harmonic model is only about $0.005 \mathrm{MeV}$. Even if it is ten times bigger for the Woods-Saxon case, the neglected term would be less than $0.1 \mathrm{MeV}$. In the last column of table 1 , we have given the Strutinsky smoothed energies $\widetilde{E}_{\mathrm{Str}}$ that were calculated in ref. ${ }^{14}$ ), and we find remarkable agreement between the two methods. Note that in ref. ${ }^{14}$ ) the Strutinsky calculations were done not by generating artificial states in the continuum 
through matrix diagonalization, but by taking account of the resonances and smoothing them.

As a second example, we also calculated $\tilde{E}_{\mathrm{sc}}$ for some axially symmetric deformed Woods-Saxon like potentials, with spin-orbit interaction included. These potentials are taken from Brack et al. ${ }^{15}$ ), and for completeness we specify them fully in ap-

TABLE 1

Smooth energy in a spherical Woods-Saxon well

\begin{tabular}{cccccccccc}
\hline$N$ & $\lambda_{\text {sc }}$ & $N \lambda_{\mathrm{sc}}$ & $E_{-3}^{0}$ & $E_{-1}^{0}$ & $E_{1}^{0}$ & $E_{-1}^{L S}$ & $E_{1}^{L S}$ & $\tilde{E}_{\mathrm{sc}}$ & $\tilde{E}_{\mathrm{Str}}$ \\
\hline 126 & -5.7323 & -722.27 & 1592.42 & -50.87 & -1.07 & 23.92 & -4.24 & -2282.43 & -2282.5 \\
184 & -4.9281 & -906.77 & 2360.52 & -61.24 & -1.47 & 30.13 & -4.97 & -3229.74 & -3230.0 \\
HO & & & 756.0 & -31.5 & 0.12 & 22.68 & -1.70 & & \\
\hline
\end{tabular}

All energies are in $\mathrm{MeV}$. The parameters of the potentials are defined in the text. The contributions of the various terms of eq. (37) to $\tilde{E}_{\mathrm{sc}}$ are shown, and in the last column the Strutinsky value ${ }^{14}$ ) is given for comparison. The last row of the table gives some representative numbers for the harmonic oscillator model of sect. 3 for comparison.

pendix C. A sample set of results for $N=82$ is shown in table 2, where the semiclassical energy $\widetilde{E}_{\mathrm{sc}}$ is computed using eqs. (24) and (25), and are compared with the corresponding Strutinsky smoothed energy $\tilde{E}_{\mathrm{Str}}$. The latter calculation was done by diagonalizing the one-body Hamiltonian in a deformed harmonic oscillator basis of twelve shells, and varying the curvature order from 6 to 16 to minimize the ambiguity of the result with respect to the smearing parameter. In making such comparisons, we take into account the truncation errors in $\widetilde{E}_{\mathrm{Str}}$ that arise from the finite basis size, and which may be substantial for a heavy nucleus. The main point of displaying table 2 is not so much the comparison with the Strutinsky method, but

TABLE 2

Smooth energy in a deformed potential $(N=82)$ for various values of the deformation parameter $c$

\begin{tabular}{ccc}
\hline$c$ & $\tilde{E}_{\text {sc }}$ & \multicolumn{1}{c}{$\tilde{E}_{\text {Str }}$} \\
\hline 1.0 & -1807.90 & $-1807.8(0.1)$ \\
1.2 & -1805.67 & $-1804.5(0.3)$ \\
1.4 & -1794.12 & $-1793.4(0.2)$ \\
1.6 & -1772.41 & $-1771.6(?)$ \\
\hline
\end{tabular}

All energies are in $\mathrm{MeV}$. The potential is defined in appendix $\mathrm{C}$. The corresponding results of the Strutinsky calculation are shown in the last column, with the associated uncertainty in the parentheses. For $c=1.6$. no plateau was found in the Strutinsky calculation, and $\tilde{E}_{\mathrm{Str}}$ is estimated at a reasonable value of the smoothing parameter. 
to demonstrate that the smooth semiclassical energy can be calculated in a straightforward manner from our formalism for realistic potential shapes. The computer time needed to complete the calculation of $\tilde{E}_{\mathrm{sc}}$ for each deformation in table 2 is about $8 \mathrm{sec}$ in a CDC-6400 computer.

In conclusion, we emphasize that the method developed in this paper for the calculation of the smooth energy generates correction terms to the usual ThomasFermi expression in a systematic manner. The formulation is only valid for potentials that are continuous functions of the coordinates. Practical calculations, which involve no free parameters, are easy to do when the potentials are given analytically, and yield unambiguous results for potentials with realistic spin-orbit strength and surface properties.

Part of this work was done when two of the authors (B.K.J. and R.K.B.) were visiting the Niels Bohr Institute last year. The authors wish to thank Prof. A. Bohr for hospitality at the institute and for discussions. The authors benefited greatly from a suggestion of Dr. H. Hoffman. For financial support, we thank the National Research Council of Canada and the Japan World Exposition Commemorative Fund.

\section{Appendix A}

The smooth energy $\tilde{E}$ (we drop the subscripts) may be written as

$$
\begin{aligned}
& \tilde{E}=\int_{0}^{\tilde{\lambda}} \tilde{g}(\varepsilon) \varepsilon \mathrm{d} \varepsilon \\
& =\tilde{\lambda} N-\int_{0}^{\tilde{\lambda}} n(\varepsilon) \mathrm{d} \varepsilon,
\end{aligned}
$$

where $n(\varepsilon)=\int_{0}^{\varepsilon} \tilde{g}(\varepsilon) \mathrm{d} \varepsilon$. In $\tilde{g}(\varepsilon)$, let us include the leading term of order $\hbar^{-3}$, and the correction terms of order $\hbar^{-1}$ as well as of order $\hbar$, so that $n(\varepsilon)$ is correct to order $\hbar$. The quantity $\tilde{\lambda}$ is determined from the equation

$$
n(\tilde{\lambda})=N \text {. }
$$

If, however, we use in eq. (A.1) an approximate Fermi energy $\tilde{\lambda}^{\prime}=\tilde{\lambda}+\delta \tilde{\lambda}$, obtained through the equation

$$
n^{\prime}\left(\tilde{\lambda^{\prime}}\right)=N
$$

where $n^{\prime}$ is correct only to order $\hbar^{-1}$, we get

$$
\tilde{E}^{\prime}=\tilde{\lambda}^{\prime} N-\int_{0}^{\tilde{\lambda}^{\prime}} n(\varepsilon) \mathrm{d} \varepsilon
$$

Note that in eq. (A.4), in the integrand of the second term we still take $n(\varepsilon)$. It is 
easy to see that

$$
\tilde{E}^{\prime}-\tilde{E}=\frac{1}{2}(\delta \tilde{\lambda})^{2} g(\tilde{\lambda})+\text { higher order terms.a }
$$

From eqs. (A.2) and (A.3), we also obtain

$$
\delta \tilde{\lambda} \approx \frac{n(\tilde{\lambda})-n^{\prime}(\tilde{\lambda})}{\tilde{g}^{\prime}(\tilde{\lambda})}
$$

Since the numerator of the above equation is of order $\hbar$, and the denominator is of order $\hbar^{-3}, \delta \tilde{\lambda}$ is of order $\hbar^{4}$. Hence $\widetilde{E}-\widetilde{E}^{\prime}$, from eq. (A.5) is of order $\hbar^{5}$. It follows that instead of using eq. (A.1), one may use eq. (A.4), and still maintain the required accuracy. In eq. (A.4), the quantity

$$
\int_{0}^{\tilde{\lambda}^{\prime}} n(\varepsilon) \mathrm{d} \varepsilon=\mathscr{L}_{\overline{\bar{\lambda}^{\prime}}}{ }^{1} Z_{\mathrm{sc}}(\beta) / \beta^{2},
$$

and since $n(\varepsilon)$ should be accurate to order $\hbar$, one should include all terms to order $\hbar$ in $Z_{\text {sc }}$.

\section{Appendix B}

The expression for $\widetilde{E}_{\mathrm{sc}}$, as given by eq. (25) of the text, may be rearranged in the form of eq. (37),

$$
\tilde{E}_{\mathrm{sc}}=\lambda_{\mathrm{sc}} N-\left(E_{-3}^{0}+E_{-1}^{0}+E_{1}^{0}\right)-\left(E_{-1}^{L S}+E_{1}^{L S}\right),
$$

where the notation is explained in the text. For spherically symmetric $U$ and $f$, the various terms of this equation reduce to the following:

$$
\begin{gathered}
E_{-3}^{0}=\frac{8}{15 \pi}\left(\frac{2 M}{\hbar^{2}}\right)^{\frac{3}{2}} \int_{0}^{r_{\mathrm{sc}}} \mathrm{d} r r^{2}\left(\lambda_{\mathrm{sc}}-U\right)^{\frac{3}{2}} \\
E_{-1}^{0}=-\frac{1}{6 \pi}\left(\frac{2 M}{\hbar^{2}}\right)^{\frac{1}{2}} \int_{0}^{r_{\mathrm{sc}}} \mathrm{d} r r^{2}\left(\lambda_{\mathrm{sc}}-U\right)^{\frac{1}{2}}\left(\frac{\mathrm{d}^{2} U}{\mathrm{~d} r^{2}}+\frac{2}{r} \frac{\mathrm{d} U}{\mathrm{~d} r}\right) \\
E_{1}^{0}=\frac{1}{120 \pi}\left(\frac{\hbar^{2}}{2 M}\right)^{\frac{1}{2}} \int_{0}^{r_{\mathrm{sc}}} \mathrm{d} r r^{2}\left(\lambda_{\mathrm{sc}}-U\right)^{-\frac{1}{2}}\left\{\frac{11}{3} \frac{1}{r^{2}} \frac{\mathrm{d}^{2} U}{\mathrm{~d} r^{2}}-\frac{5}{12} \frac{\mathrm{d}^{4} U}{\mathrm{~d} r^{4}}\right. \\
\left.+\frac{7}{6} \frac{1}{r}\left(\frac{\mathrm{d}^{2} U}{\mathrm{~d} r^{2}}\right)^{2}\left(\frac{\mathrm{d} U}{\mathrm{~d} r}\right)^{-1}+\frac{7}{6} \frac{\mathrm{d}^{2} U}{\mathrm{~d} r^{2}} \frac{\mathrm{d}^{3} U}{\mathrm{~d} r^{3}}\left(\frac{\mathrm{d} U}{\mathrm{~d} r}\right)^{-1}-\frac{7}{12}\left(\frac{\mathrm{d}^{2} U}{\mathrm{~d} r^{2}}\right)^{3}\left(\frac{\mathrm{d} U}{\mathrm{~d} r}\right)^{-2}\right\} \\
E_{-1}^{L S}=\frac{2 \kappa^{2}}{3 \pi}\left(\frac{2 M}{\hbar^{2}}\right)^{\frac{1}{2}} \int_{0}^{r_{\mathrm{sc}}} \mathrm{d} r r^{2}\left(\lambda_{\mathrm{sc}}-U\right)^{\frac{3}{2}}\left(\frac{\mathrm{d} f}{\mathrm{~d} r}\right)^{2} \\
E_{1}^{L S}=-\frac{\kappa^{2}}{24 \pi}\left(\frac{\hbar^{2}}{2 M}\right)^{\frac{1}{2}} \int_{0}^{r_{\mathrm{sc}}} \mathrm{d} r r^{2}\left(\lambda_{\mathrm{sc}}-U\right)^{-\frac{1}{2}}\left(\frac{\mathrm{d} f}{\mathrm{~d} r}\right)^{2} \frac{\mathrm{d}^{2} U}{\mathrm{~d} r^{2}}
\end{gathered}
$$




$$
\begin{aligned}
& +\frac{1}{3 \pi}\left(\frac{\hbar^{2}}{2 M}\right)^{\frac{1}{2}} \int_{0}^{r_{\mathrm{sc}}} \mathrm{d} r r^{2}\left(\lambda_{\mathrm{sc}}-U\right)^{\frac{1}{2}}\left[\kappa ^ { 2 } \left\{\frac{1}{2} \frac{\mathrm{d} f}{\mathrm{~d} r} \frac{\mathrm{d}^{3} f}{\mathrm{~d} r^{3}}-\frac{1}{r} \frac{\mathrm{d} f}{\mathrm{~d} r} \frac{\mathrm{d}^{2} f}{\mathrm{~d} r^{2}}\right.\right. \\
& \left.\left.-\frac{2}{r^{2}}\left(\frac{\mathrm{d} f}{\mathrm{~d} r}\right)^{2}\right\}-\kappa^{3} \frac{1}{r}\left(\frac{\mathrm{d} f}{\mathrm{~d} r}\right)^{3}+\frac{\kappa^{4}}{2}\left(\frac{\mathrm{d} f}{\mathrm{~d} r}\right)^{4}\right] .
\end{aligned}
$$

In the spherical case, therefore, evaluation of only one-dimensional integrals are involved.

For axially symmetric deformed $U$ and $f$, it is best to use cylindrical coordinates in evaluating eq. (25). The resulting expression for $\widetilde{E}_{\mathrm{sc}}$ is rather long and cumbersome, but is straight-forward to work out and we do not write it down. All the integrals in this case are two-dimensional.

\section{Appendix C}

The deformed potential shapes were taken from ref. ${ }^{15}$ ) as defined in their eqs. (VII, $5 ; 20 ; 21)$; and (31). The central part of the potential $U$ is written in terms of reduced cylindrical variables $u$ and $v$, defined in terms of the cylindrical coordinates $\rho$ and $\mathscr{Z}$ as

$$
\rho=c R v, \quad \mathscr{Z}=c R u ;
$$

where $c$ is a dimensionless parameter and $R$ an effective radius. Defining a quantity $\pi$ in terms of these variables,

$$
\pi=v^{2}+\left(u^{2}-1\right)\left(A+B u^{2}\right),
$$

with $A=c^{-3}-\frac{1}{10}(c-1)$ and $B=\frac{1}{2}(c-1)$, one writes the potential $U$ as

$$
U=-V_{0} /\left(1+\mathrm{e}^{l(u, v)}\right) \text {. }
$$

In the above equation,

$$
l(u, v)=\frac{c R}{a} \frac{\pi}{|\nabla \pi|}
$$

where the gradient is taken with respect to $u$ and $v$. For the central part, for $N=82$, we take $V_{0}=49.355 \mathrm{MeV}, R=6.433 \mathrm{fm}$ and $a=0.66 \mathrm{fm}$. For the spin-orbit potential $V_{L S}$, as defined in eq. (15), the form factor $f$ is given by

$$
f=\left[1+\mathrm{e}^{l_{\mathrm{s}}(u, v)}\right]^{-1}, \quad l_{\mathrm{s}}(u, v)=\frac{c R_{\mathrm{s}}}{a_{\mathrm{s}}} \frac{\pi}{|\nabla \pi|},
$$

and we take, for $N=82 ; \kappa=0.5784, R_{\mathrm{s}}=5.780 \mathrm{fm}$ and $a_{\mathrm{s}}=0.55 \mathrm{fm}$. The numerical values for the parameters of the potential are taken from ref. ${ }^{16}$ ). Note that for $c=1$, 
$U$ and $f$ become spherically symmetric. In this case,

$$
U=-V_{0} /\left(1+\mathrm{e}^{\left(r^{2}-R^{2}\right) / 2 r a}\right),
$$

so that $R$ is the half-fall-off radius and $a$ determines the skin thickness. The surface properties of this potential are similar to that of a Woods-Saxon one.

\section{References}

1) V. M. Strutinsky, Nucl. Phys. A95 (1967) 420

2) V. M. Strutinsky, Nucl. Phys. A122 (1968) 1

3) E. Wigner, Phys. Rev. 40 (1932) 749

4) J. G. Kirkwood, Phys. Rev. 44 (1933) 31

5) M. Bolsterli, E. O. Fiset, J. R. Nix and J. L. Norton, Phys. Rev. C5 (1972) 1050

6) M. Brack and H. C. Pauli, Nucl. Phys. A207 (1973) 401

7) A. Sobiczewski et al., to be published

8) B. K. Jennings, R. K. Bhaduri and M. Brack, Phys. Rev. Lett. 34 (1975) 228

9) R. K. Bhaduri and C. K. Ross, Phys. Rev. Lett. 27 (1971) 606

10) B. K. Jennings, Ann. of Phys. 84 (1974) 1

11) B. K. Jennings and R. K. Bhaduri, Nucl. Phys. A237 (1975) 149

12) G. E. Uhlenbeck and E. Beth, Physica 3 (1936) 729

13) J. Blomquist and S. Wahlborn, Ark. Fys. 16 (1960) 545

14) C. K. Ross and R. K. Bhaduri, Nucl. Phys. A188 (1972) 566

15) M. Brack, J. Damgaard, A. S. Jensen, H. C. Pauli, V. M. Strutinsky and C. Y. Wong, Rev. Mod. Phys. 44 (1972) 320

16) H. C. Pauli, Phys. Reports 7 (1973) 35 